\title{
Effectiveness and Safety of the Clareon Monofocal Intraocular Lens: Outcomes from a 12-Month Single-Arm Clinical Study in a Large Sample
}

\author{
Robert Lehmann (D) \\ Andrew Maxwell ${ }^{2}$ \\ David M Lubeck ${ }^{3}$ \\ Raymond Fong ${ }^{4}$ \\ Thomas R Walters (D) ${ }^{5}$ \\ Anna Fakadej ${ }^{6}$ \\ 'Lehmann Eye Center, Nacogdoches, TX, \\ USA; ${ }^{2}$ California Eye Institute, Fresno, \\ CA, USA; ${ }^{3}$ Arbor Centers for EyeCare, \\ Homewood, IL, USA; ${ }^{4}$ Manhattan Eye, \\ Ear, and Throat Hospital, Lenox Hill \\ Hospital, New York, NY, USA; ${ }^{5}$ Texan \\ Eye, Austin, TX, USA; ${ }^{6}$ Carolina Eye \\ Associates, PA, Southern Pines, NC, USA
}

Correspondence: Robert Lehmann Lehmann Eye Center, Nacogdoches, TX, USA

Tel + I 936-569-8278

Email doctorlehmann@me.com
Purpose: This study assessed effectiveness and safety of the novel Clareon intraocular lens (IOL; model SY60CL; Alcon Vision LLC).

Patients and Methods: This was a prospective, single-arm, unmasked clinical trial at 16 investigative clinical sites in the United States. Included were adults $\geq 22$ years who required cataract extraction by phacoemulsification. Following phacoemulsification, 350 subjects received SY60CL IOL unilaterally; 342 completed the study. Monocular best corrected distance visual acuity (CDVA) and uncorrected distance visual acuity (UDVA) were evaluated. The primary effectiveness endpoint was the percentage of subjects with CDVA $\leq 0.3$ $\log$ MAR at month 12. Safety was assessed by monitoring adverse events (AEs). Visual acuity and safety outcomes were compared with historical safety and performance endpoint (SPE) rates.

Results: At 12 months post-implantation, 99.7\% of subjects receiving the SY60CL IOL achieved monocular CDVA $\leq 0.3$ logMAR (primary effectiveness endpoint; 1 -sided 95\% upper confidence limit >SPE rate); $99.7 \%$ and $86.8 \%$ of subjects achieved monocular CDVA of $\leq 0.34$ (20/40 Snellen or better) and $\leq 0.04 \operatorname{logMAR}$ (20/20 Snellen or better), respectively. At 12 months, $>95 \%$ of subjects achieved mean monocular UDVA $\leq 0.3 \log$ MAR; $97.1 \%$ and $57.6 \%$ of subjects achieved monocular CDVA of $\leq 0.34$ and $\leq 0.04 \operatorname{logMAR}$, respectively. Mean monocular CDVA and UDVA were -0.05 and $0.04 \operatorname{logMAR}$, respectively. AEs were within SPE limits. The most common nonserious ocular AE was posterior capsule opacification (5.4\%). Serious AEs were $<1 \%$, and no serious ocular AEs were assessed as related to the device. There were no observations for IOL glistenings at 12 months.

Conclusion: Results of this study supported effectiveness and safety of the SY60CL IOL. Visual acuity outcomes with the SY60CL IOL exceeded the SPE rates for monocular CDVA and AEs were within the limit of historic SPE rates. (Model number SY60WF is the Clareon lens approved by the FDA.)

Keywords: glistenings, posterior capsule opacification, visual acuity, cystoid macular edema, dysphotopsia

Cataract is the leading cause of blindness worldwide; standard-of-care treatment is removal of the cloudy natural crystalline lens and implantation of an intraocular lens (IOL). ${ }^{1,2}$ In addition to the restored visual acuity (VA) provided by this treatment, advances in IOL design and materials can potentially reduce the rate of adverse events (AEs) and photic phenomena, leading to further improvements in visual function and patient satisfaction. 
The Clareon ${ }^{\circledR}$ IOL (model SY60CL; Alcon Vision LLC, Fort Worth, TX, USA) is a novel monofocal aspheric hydrophobic lens composed of a flexible acrylic material (PEA/HEMA copolymer) with high refractive index (Clareon model number SY60WF will be commercially available). This ultraviolet (UV)-absorbing and blue lightfiltering lens uses a different material but has the same optical design as the AcrySof ${ }^{\circledR}$ Natural IQ IOL (model SN60WF; Alcon Vision LLC). It is a foldable, single-piece IOL for implantation in the capsular bag in the posterior chamber during cataract surgery.

Different IOL materials can exhibit different lens artifacts such as microvacuoles (ie, glistenings). ${ }^{3,4}$ Both glistening and surface light scattering were reported to be more severe in hydrophobic acrylic IOLs compared with silicone and polymethyl methacrylate IOLs. ${ }^{4}$ A prospective randomized study in 273 patients reported that approximately $40 \%$ to $68 \%$ of patients developed glistenings, depending on the lens type, 2 years postimplantation. ${ }^{5}$ The SY60CL IOL material was designed to enhance clarity and has demonstrated among the lowest levels of surface haze, surface roughness, and glistenings compared with commercially available hydrophobic acrylic IOLs. ${ }^{6}$ In this in vitro study, Clareon model CNA0T0 had significantly lower microvacuole density and size compared with Tecnis model ZCB00 (Johnson \& Johnson Vision, Santa Ana, CA, USA), Tecnis OptiBlue ZCB00V, or Vivinex XY1 (Hoya Surgical Optics, Inc., Chino Hills, CA, USA) lenses $(P<0.001)$. Similar microvacuole density and size were reported for the Clareon IOL, Eternity W-60 (Santen, Inc., Osaka, Japan), and enVista MX60 (Bausch \& Lomb, Rochester, NY, USA) lenses $(P>0.4)$, which had higher water content. ${ }^{6}$ Recently, these in vitro data were corroborated by clinical studies. No glistenings or surface haze were reported in eyes with the Clareon IOL 1 year $(n=110)$ and 9 years $(n=20)$ postimplantation. ${ }^{7}$ A randomized controlled trial reported minimal glistenings (median grade 0 on an 8-point scale) in subjects implanted with Clareon $(\mathrm{n}=68)$ or Tecnis $(\mathrm{n}=68)$ IOLs at 1 year post-implantation, with no significant difference between the 2 groups $(P=0.2)$.

Several studies demonstrated that IOL material and design can have a significant effect on posterior capsule opacification (PCO) and neodymium-doped yttrium aluminum garnet (Nd:YAG) capsulotomy rates. ${ }^{9-12}$ AcrySof IOLs were reported to have lower Nd:YAG capsulotomy rates compared with round-edged silicone IOLs $(P=0.001) .{ }^{9}$ A meta-analysis of randomized controlled trials reported that PCO rates were significantly lower for hydrophobic acrylic versus hydrophilic acrylic IOLs at 1and 2-year follow-up ( $P=0.0002$ and $P<0.00001$, respectively). Additionally, hydrophobic acrylic IOLs were associated with significantly lower rates of Nd:YAG capsulotomy than hydrophilic IOLs $(P<0.00001) .^{10}$

The purpose of this study was to assess effectiveness and safety of the novel SY60CL material in a clinical trial in adults who underwent cataract surgery and to compare VA and safety outcomes of the SY60CL IOL with historical safety and performance endpoint (SPE) rates. Specifically, we evaluated the effects of the SY60CL material on glistenings, cystoid macular edema (CME), and PCO.

\section{Methods}

\section{Study Design}

This was a prospective, multicenter (16 sites in the United States), single-arm, unmasked clinical trial conducted from July 2017 to February 2019 (clinicaltrials.gov registration: NCT03170154). A sample size of 350 subjects was planned so that $\geq 300$ evaluable eyes with successful implantation would be available for analysis. With 300 evaluable eyes, the probability of demonstrating that $\geq 90 \%$ of eyes had monocular CDVA of $0.3 \log$ MAR or better at 12 months was $99 \%$. Included in the study were adults aged $\geq 22$ years who required cataract extraction by phacoemulsification in at least 1 qualifying eye and for whom postoperative emmetropia was planned $( \pm 0.5$ D spherical equivalent). This clinical trial was conducted under Investigational Device Exemption G170112 approved by the FDA on May 31, 2017.

Key exclusion criteria were disease or pathology other than cataract expected to reduce potential postoperative best corrected distance visual acuity (CDVA) to worse than $0.30 \log \mathrm{MAR}$, previous corneal surgery, rubella or traumatic cataract, ocular trauma or previous refractive surgery, or use of agents (eg, $\alpha_{1}$-antagonist) that would require mechanical or surgical manipulation to enlarge the pupil. Subjects were discontinued from the study during the surgery if there were intraoperative complications requiring additional procedures or leading to the surgeon's inability to place the IOL in the capsular bag.

Subjects attended 7 study visits including a preoperative screening visit; the operative visit; and postoperative visits on day 1 , week 1 , month 1 , month 6 , and month 12. Unscheduled visits were conducted as needed. 


\section{Surgical Procedure}

Cataracts were removed by phacoemulsification using a surgeon's preferred methodology, and subjects received a SY60CL monofocal IOL unilaterally using a Monarch ${ }^{\circledR}$ III D cartridge and handpiece. Routine operative medications were used per standard surgical protocol at individual sites. Surgical manipulation to enlarge the pupil was not allowed. The reference provisional A-constant was 119.1. The SY60CL IOL is a 1-piece aspheric hydrophobic acrylic monofocal lens made with a UV-absorbing and blue lightfiltering acrylate/methacrylate copolymer. SY60CL was the model designated for clinical investigation. Model number SY60WF is the Clareon lens that has been approved by the FDA. Other lens characteristics include Stableforce ${ }^{\circledR}$ modified-L haptics (Alcon Vision LLC), a 6.0-mm optic diameter, and 13.0-mm overall length. Representative images of the SY60CL IOL include an in vitro image taken with a Nikon Eclipse Ti-U inverted microscope at $15 \times$ magnification (Figure 1A) and a slit lamp image taken at study visit 3 (month 1) using retroillumination at $10 \times$ magnification, with a flash intensity of $75 \%$ and an aperture setting of 4 under dilated pupil conditions (Figure 1B). Toric axis markings were incorporated on the non-toric SY60CL monofocal IOL for evaluation of rotational stability and are not part of the commercially available lens.

\section{Effectiveness and Safety Assessments}

The primary effectiveness endpoint was the percentage of allimplanted subjects with CDVA $\leq 0.3 \log$ MAR at month 12 . Refraction was performed using a phoropter or trial frames at $4 \mathrm{~m}$, and the spherical component was increased or decreased in $0.25 \mathrm{D}$ steps with the aim of achieving maximum plus diopter spherical correction for maximum visual acuity. If needed, cylinder was refined using the cross-cylinder technique. One-sided exact 95\% upper confidence limit was determined and study success was concluded if it was $0.3 \log$ MAR or better compared with the SPE rate of $92.5 \%$ for the allimplanted analysis set. VA was measured using the fast Early Treatment Diabetic Retinopathy Study (ETDRS) method. ${ }^{13}$ Effectiveness was also assessed by monocular CDVA and uncorrected distance visual acuity (UDVA) at $4 \mathrm{~m}$. CDVA and UDVA data were summarized using descriptive statistics.

Safety was assessed by monitoring AEs, including secondary surgical interventions. PCO and glistenings were assessed during slit-lamp examination. If present, $\mathrm{PCO}$ was graded as clinically non-significant (ie, early development of PCO such as fibrosis and proliferation of lens epithelial cells; no decrease in visual acuity or increase in glare), clinically significant (increased PCO with early visual acuity changes that did not require posterior capsulotomy), or clinically significant requiring YAG (PCO adversely affecting visual acuity and requiring posterior capsulotomy). For evaluation of glistenings, photographic examples were provided as a reference aid. Negative and positive dysphotopsia were evaluated during routine assessment of AEs. AEs were summarized using descriptive statistics. The one-sided exact 95\% lower confidence level for incidence rates of AEs observed for the study eyes was compared with the cumulative and persistent $\mathrm{AE}$ SPE rates as reported in EN ISO 11979-7:2014. ${ }^{14}$

\section{Statement of Ethics}

This study was conducted in accordance with the principles of the Declaration of Helsinki and in compliance with Good Clinical Practice, ISO 11979-7:2014, ISO 14155:2011, and code of federal regulations. Approval was obtained from the Sterling Institutional review board
A

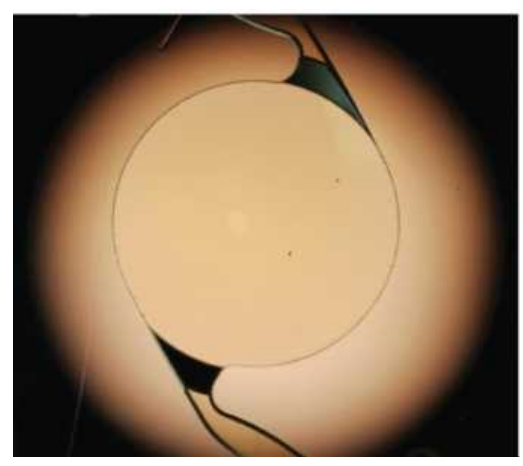

B

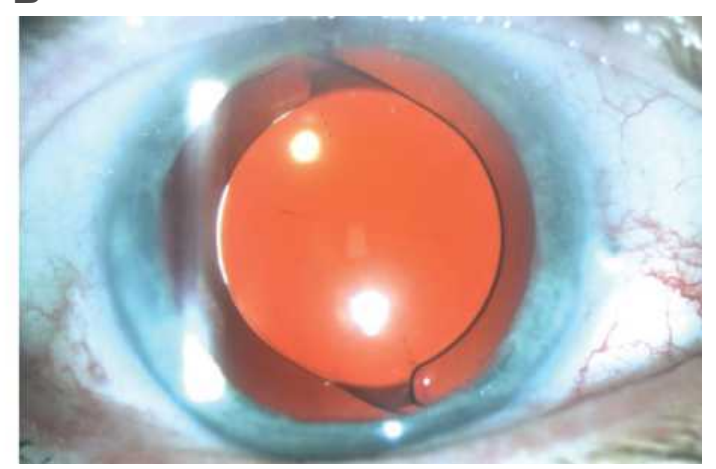

Figure I Representative SY60CL IOL images. ${ }^{\text {aSY } 60 C L ~ I O L ~ w i t h ~ t h e ~ N i k o n ~ E c l i p s e ~ T i-U ~ i n v e r t e d ~ m i c r o s c o p e ~ a t ~ I 5 \times ~ m a g n i f i c a t i o n ~(A) ~ a n d ~ s l i t ~ l a m p ~ p h o t o g r a p h y ~ u s i n g ~}$ retroillumination captured under pupil dilation conditions at 10x magnification, with a flash intensity of $75 \%$ and an aperture setting of 4 (B). ${ }^{a} S Y 60 C L$ was the designated lens model for clinical investigation; model number SY60WF is the Clareon lens that has been approved by the United States Food and Drug Administration. 
(IRB). Because none of the investigators who participated were at sites with associated institutional IRBs, a central IRB was contracted to perform ethics oversight. Sterling IRB went through a rigorous vendor approval process that included routine quality assurance audits per Alcon's procurement requirements. Subjects provided valid written informed consent before any screening or trial-related procedures.

\section{Results}

\section{Subjects}

Of the 376 subjects enrolled, 26 were excluded from the study before implantation because of screen failure, and 350 received the SY60CL IOL (Figure 2). Of the 350 subjects, 342 completed the study. Eight subjects were discontinued from the study after implantation: 4 were lost to follow-up, 2 withdrew, and 2 subjects died (deaths were not related to the study device or procedure).

Most subjects were white $(273 / 350,78.0 \%)$ and were $\geq 65$ years old $(281 / 350,80.3 \%$; Table 1$)$, with a mean age of 69.7 years. More than half of subjects were female (213/350, 61\%). Mean baseline CDVA was $0.19 \pm 0.21$ logMAR, and mean baseline corneal astigmatism was $0.65 \pm 0.42 \mathrm{D}$ (Table 1).

\section{Visual Acuity Outcomes}

At month 12, 99.7\% of subjects achieved CDVA of 0.3 $\operatorname{logMAR}$ or better, and the one-sided 95\% upper confidence limit of $99.99 \%$ was greater than the SPE rate of $92.5 \%$. Additionally, $81.0 \%$ of subjects achieved CDVA of $0.0 \log$ MAR or better at month 12 .

Mean monocular CDVA was -0.01 and $-0.05 \log$ MAR at week 1 and month 12, respectively (Figure 3A). The cumulative distribution of monocular CDVA in subjects was similar at most postoperative visits. Monocular CDVA $\leq 0.34 \operatorname{logMAR}$ (20/40 Snellen or better) was achieved by $98.6 \%$ and $99.7 \%$ of subjects at week 1 and month 12 , respectively (Figure 3B). Monocular CDVA $\leq 0.04$ logMAR (20/20 Snellen or better) was achieved by $77.4 \%$ and $86.8 \%$ of subjects at week 1 and month 12 , respectively (Figure 3B). One subject had CDVA of $0.44 \log$ MAR at month 12 that was related to PCO. After Nd:YAG, the subject achieved CDVA of $0.1 \log$ MAR (20/25 Snellen).

Mean monocular UDVA was 0.09 and $0.04 \log$ MAR at week 1 and month 12, respectively (Figure 4A). More than $95 \%$ of subjects achieved monocular UDVA of 0.3 $\log$ MAR or better. Monocular UDVA $\leq 0.34$ logMAR (20/40 Snellen or better) was achieved by $94.0 \%$ and $97.1 \%$ of subjects at week 1 and month 12 , respectively (Figure 4B). Monocular UDVA $\leq 0.04 \log$ MAR (20/20

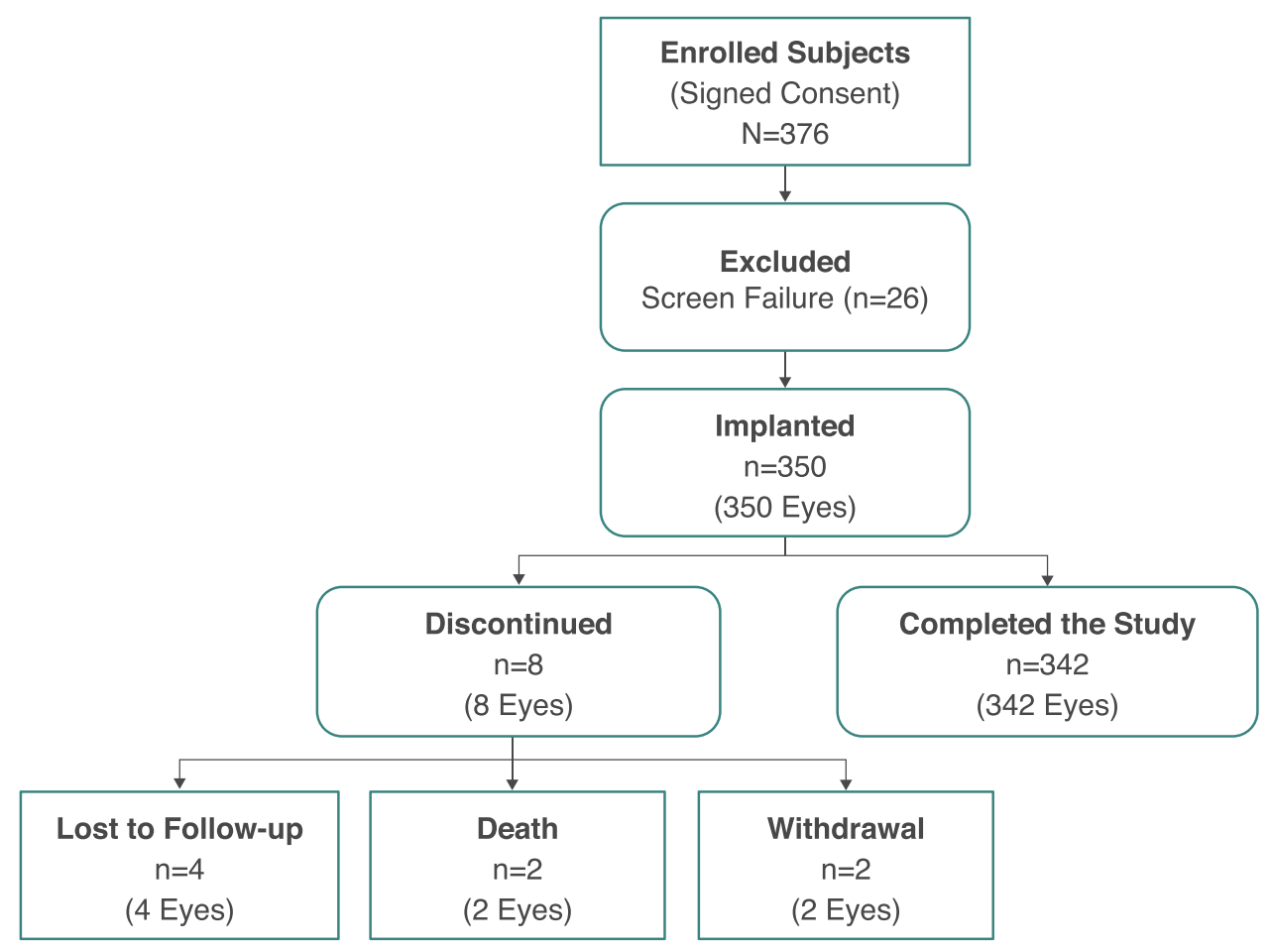

Figure 2 Subject disposition. 
Table I Subject Demographics and Baseline Characteristics (AllImplanted Analysis Set)

\begin{tabular}{|c|c|}
\hline Parameter & SY60CL IOL $(n=350)$ \\
\hline \multicolumn{2}{|l|}{ Age, years } \\
\hline Mean \pm SD & $69.7 \pm 6.44$ \\
\hline Range & $45-86$ \\
\hline \multicolumn{2}{|l|}{ Age group, years, $n(\%)$} \\
\hline$<65$ & $69(20)$ \\
\hline$\geq 65$ & $28 \mid(80)$ \\
\hline \multicolumn{2}{|l|}{ Sex, n (\%) } \\
\hline Female & $213(61)$ \\
\hline Male & 137 (39) \\
\hline \multicolumn{2}{|l|}{ Race, n (\%) } \\
\hline White & $273(78)$ \\
\hline Black or African American & $36(10)$ \\
\hline Asian & $29(8.3)$ \\
\hline Native Hawaiian or other Pacific Islander & $\mathrm{I}(0.3)$ \\
\hline Other & II (3.I) \\
\hline \multicolumn{2}{|l|}{ Monocular CDVA, logMAR } \\
\hline Mean \pm SD & $0.191 \pm 0.206$ \\
\hline Range & -0.18 to 1.70 \\
\hline \multicolumn{2}{|l|}{ Axial length, mm } \\
\hline Mean \pm SD & $23.9 \pm 0.96$ \\
\hline Range & $21.3-27.1$ \\
\hline \multicolumn{2}{|l|}{ Axial length category, $n(\%)$} \\
\hline Short $(<21 \mathrm{~mm})$ & 0 \\
\hline Medium $(2 \mathrm{I}-26 \mathrm{~mm})$ & $343(98)$ \\
\hline Long (>26 mm) & $7(2)$ \\
\hline \multicolumn{2}{|l|}{ Corneal astigmatism, D } \\
\hline Mean \pm SD & $0.647 \pm 0.418$ \\
\hline Range & $0.0-3.15$ \\
\hline
\end{tabular}

Abbreviation: CDVA, best corrected distance visual acuity.

Snellen or better) was achieved by $45.8 \%$ and $57.6 \%$ of subjects at week 1 and month 12, respectively (Figure 4B).

\section{Mean Manifest Refraction Spherical Equivalent}

At the preoperative visit, mean manifest refraction spherical equivalent (MRSE) was $-0.39 \mathrm{D}$, and median MRSE was $0.0 \mathrm{D} ; 55.7 \%$ of subjects had MRSE $>1$ D. Subjects achieved mean MRSE of -0.03 and $0.04 \mathrm{D}$ at week 1 and month 12, respectively (Figure 5A). Median MRSE was $0.0 \mathrm{D}$ at all visits. Cumulative distribution of absolute MRSE in subjects was stable at visits between week 1 and month 12. Absolute MRSE was within $1.0 \mathrm{D}$ in $98.9 \%$ $(345 / 349)$ and $98.8 \%(338 / 342)$ of subjects at week 1 and month 12, respectively. Absolute MRSE was within 0.5 $\mathrm{D}$ in $85.7 \%$ (299/349) and $85.1 \%(291 / 342)$ of subjects at week 1 and month 12, respectively (Figure 5B).

Mean target residual refractive error was $-0.12 \mathrm{D}$. At week 1, mean prediction error was $0.09 \mathrm{D} ; 98.6 \%$ of subjects had absolute prediction error $\leq 1.0 \mathrm{D}$, and $79.1 \%$ had absolute prediction error $\leq 0.5 \mathrm{D}$. At month 12 , mean prediction error was $0.17 \mathrm{D} ; 97.7 \%$ of subjects had absolute prediction error $\leq 1.0 \mathrm{D}$, and $76.3 \%$ had absolute prediction error $\leq 0.5 \mathrm{D}$.

\section{Safety Outcomes}

The rate of cumulative and persistent AEs was within the limit of the SPE rates based on the 1-sided exact $95 \%$ lower confidence level. The most frequently reported nonserious ocular AEs included PCO (19/350, 5.4\%) and increased intraocular pressure $(17 / 350,4.9 \%)$. Other ocular AEs occurred at rates of $\leq 2.3 \%$ (Table 2). Posterior capsulotomy was required in 16 subjects $(4.6 \%) ; 6$ of those subjects underwent $\mathrm{Nd}: \mathrm{YAG}$ at a single site (total number of subjects enrolled at that site was 20); the rate of posterior capsulotomy among the remaining sites was 10 / $330(3.0 \%)$.

Rates of serious ocular AEs were $<1 \%$ (Table 3) and included CME $(3 / 350,0.9 \%)$ and retinal laser coagulation, retinal tear, and vitrectomy $(2 / 350,0.6 \%$ each). One subject had a moderately severe herpetic lesion and received ophthalmologic treatment, including anti-viral medication and Prokera placement. All serious ocular AEs were assessed by the investigator as not related to the device. Serious AEs assessed as related to the procedure included retinal tear and retinal laser coagulation, corneal epithelial repair with amniotic membrane graft and superficial punctate keratitis, full-thickness macular hole, and CME ( $\mathrm{n}=1$ for each).

All adverse events related to the device were mild and resolved (visual impairment including negative or positive dysphotopsia; $3 / 350,0.9 \%$ ). There were no observations of IOL glistenings at 12 months. Additionally, there were no reports of IOL surface haze, scratches, or cracks.

\section{Discussion}

The SY60CL IOL evaluated in this study was based on the AcrySof platform and uses a novel hydrophobic acrylic polymer lens material designed to enhance clarity. ${ }^{15,16}$ The results of this study indicate that $>99 \%$ of subjects who received the SY60CL IOL achieved monocular CDVA of $0.3 \log$ MAR or better 12 months 
A
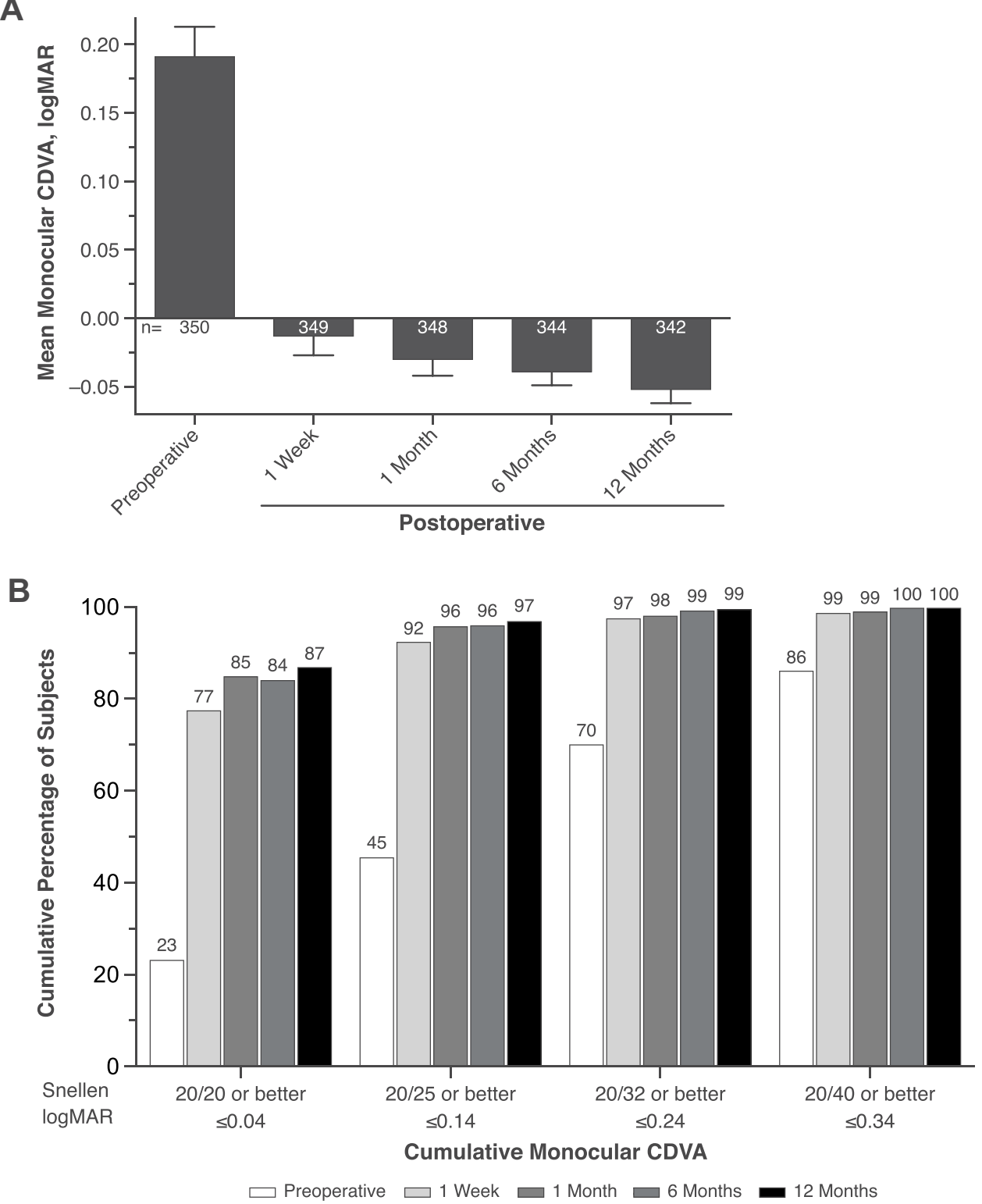

Figure 3 Mean monocular CDVA at $4 \mathrm{~m}$ (A) and cumulative distribution of monocular CDVA (B) in subjects who received SY60CL IOL. Error bars represent 95\% Cls. Abbreviations: CDVA, best corrected distance visual acuity; IOL, intraocular lens.

after implantation (one-sided exact 95\% upper confidence limit, $99.99 \%$ ), exceeding the current performance standards for monofocal IOLs. At month $12,>95 \%$ of subjects achieved monocular UDVA of $0.3 \log$ MAR. Additionally, $87 \%$ of subjects had a CDVA of $20 / 20$ or better, and $97 \%$ of subjects had functional UDVA of 20 / 40 or better at 12 months. The cumulative and persistent AE rate was within the limits of the SPE rates, and no serious ocular AEs or secondary surgical interventions were assessed as related to the device.

The results of this study are consistent with an interim report that demonstrated improved CDVA at week 1 through month 6 and no unanticipated AEs. ${ }^{17}$ Also consistent with the 6-month interim results, there were no observations of glistenings or surface haze reported for the SY60CL IOL at 12 months.

Glistenings have been linked to IOL properties, such as IOL material, manufacturing process, and temperature fluctuations, and have been reported to be most common in hydrophobic acrylic IOLs. ${ }^{3,4,18}$ In a prospective study with 273 subjects who received 7 types of IOLs, the AcrySof IOL had a higher percentage of glistenings compared with other IOLs up to 2 years after surgery. ${ }^{5}$ Novel IOL materials, such as SY60CL, have been designed to 

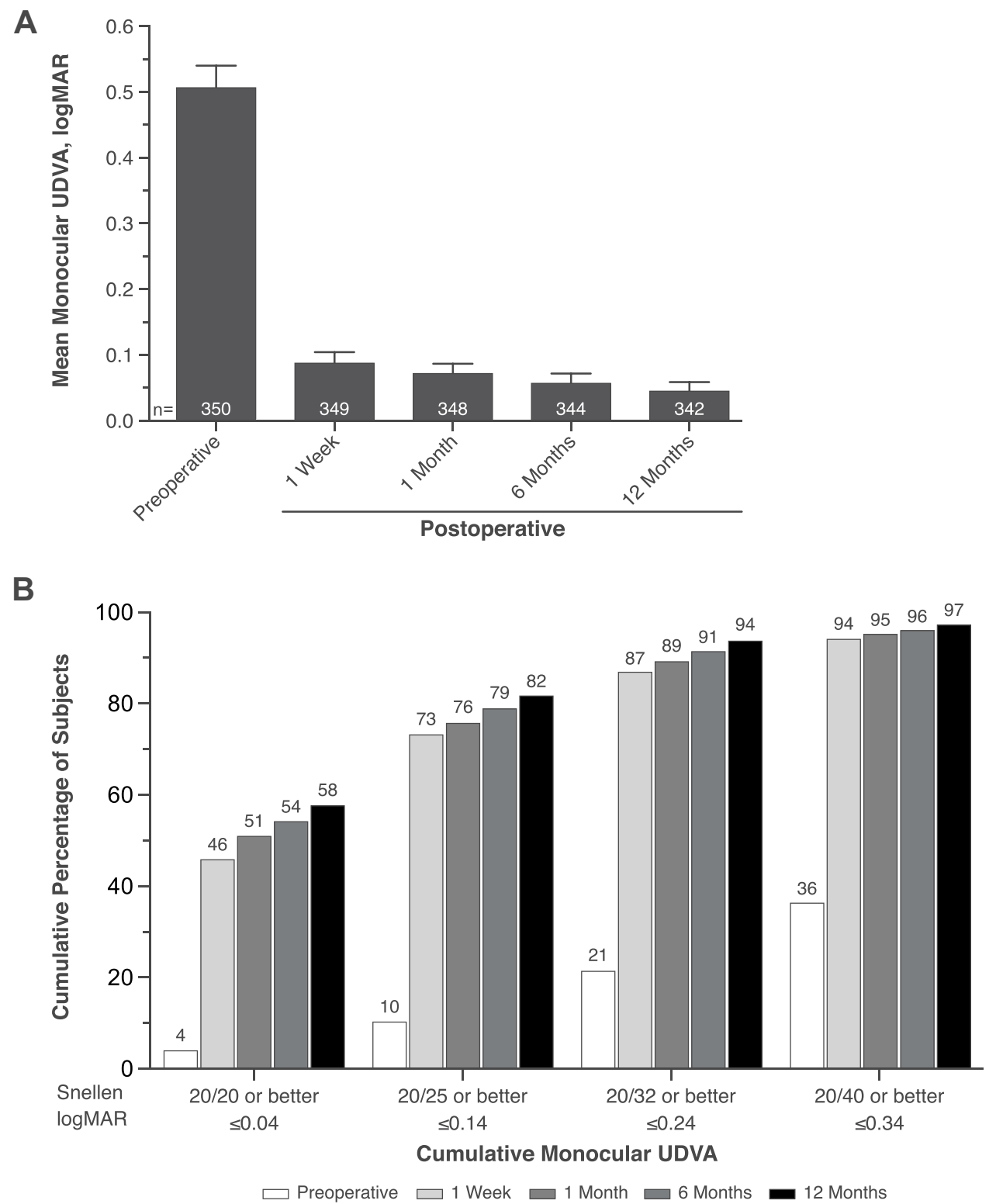

Figure 4 Mean monocular UDVA at $4 \mathrm{~m}$ (A) and cumulative distribution of monocular UDVA (B) in subjects who received SY60CL IOL. Error bars represent $95 \%$ Cls. Abbreviations: IOL, intraocular lens; UDVA, uncorrected distance visual acuity.

reduce glistenings. ${ }^{3}$ In an in vitro evaluation, the SY60CL IOL had among the lowest levels of surface haze and glistenings compared with Tecnis ZCB00, Tecnis OptiBlue ZCB00V, Eternity W-60, Vivinex XY1, or enVista MX60. ${ }^{6}$ Additionally, recent clinical studies reported no or minimal glistenings in subjects who received the SY60CL IOL. ${ }^{7}$ In the current clinical study, no IOL glistenings were reported at any visit; additional long-term clinical studies are needed to confirm the low rates of glistenings in subjects receiving Clareon IOLs and to further address the effects of the material on clarity of vision.
Estimates of PCO and Nd:YAG rates vary depending on the type of lens and experimental design. A 1998 metaanalysis reported that overall pooled estimates of PCO incidence were $11.8 \%$ at year $1,20.7 \%$ at year 3, and $28.4 \%$ at year 5 postoperatively. ${ }^{19}$ More recently, analysis of postmortem human eyes showed that the Nd:YAG capsulotomy rate ranged between $0.9 \%$ and $17.1 \%$ for modern IOLs. ${ }^{20}$ A prospective study reported that the incidence of residual capsule opacity was 44/194 (22.7\%); however, only 5 cases $(3.5 \%)$ were considered "visually significant" at the 6-week postoperative visit. ${ }^{21} \mathrm{~A}$ recent meta-analysis of 17,691 eyes in Finland reported that overall Nd:YAG 
A

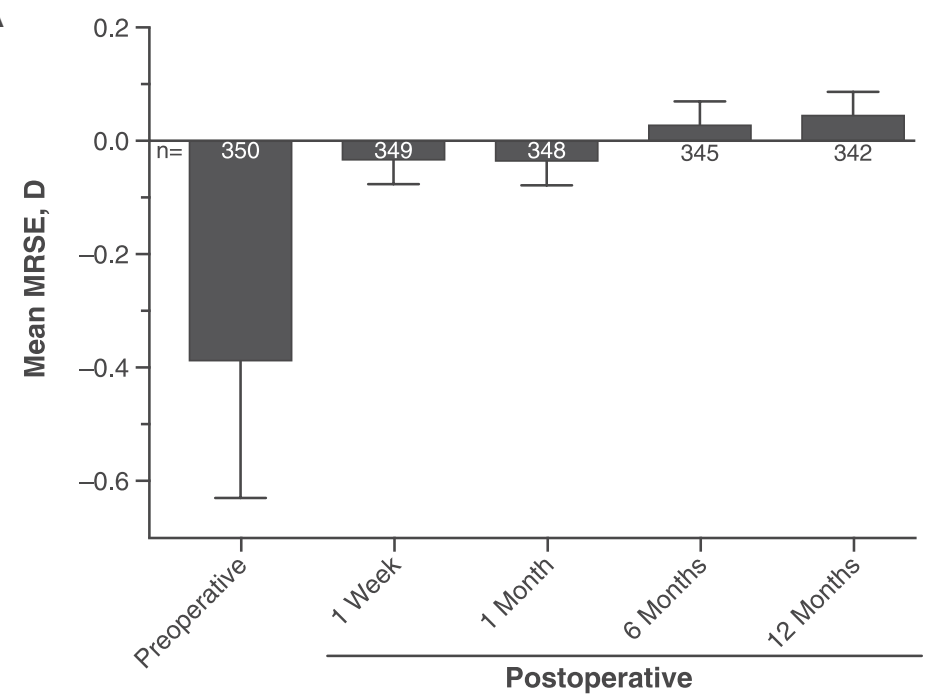

B

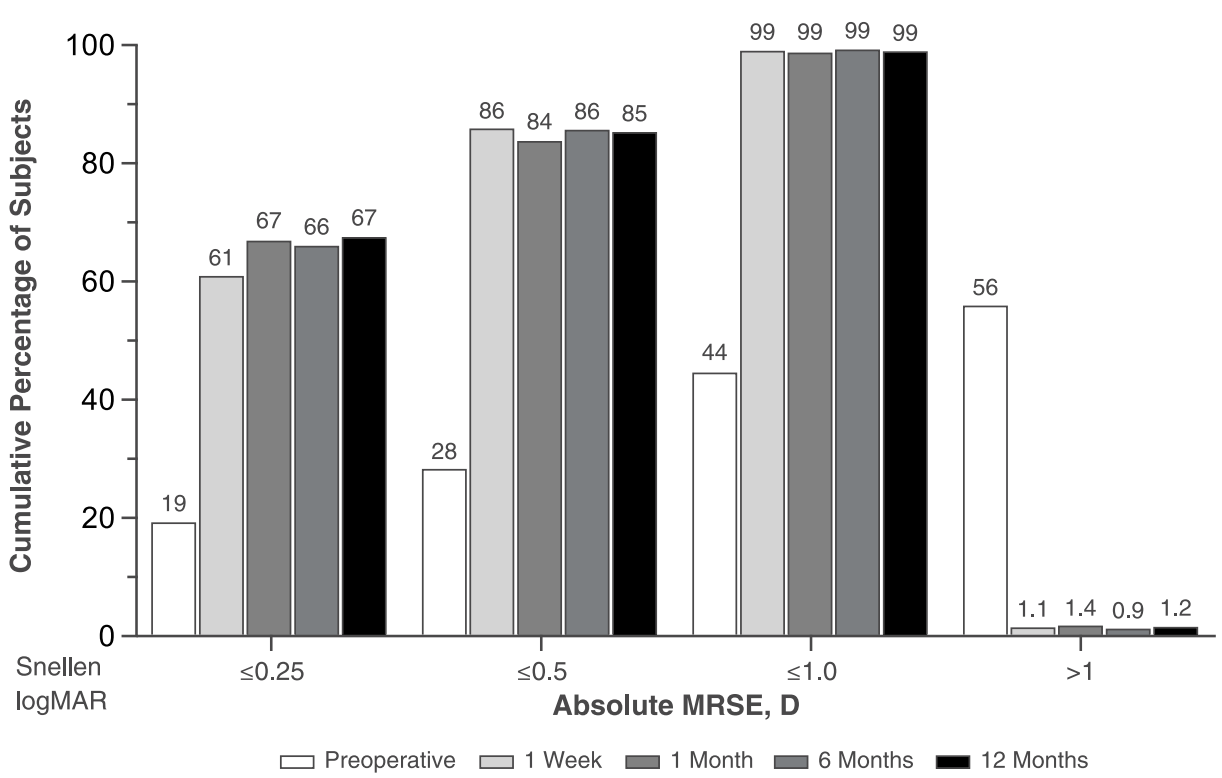

Figure 5 Mean MRSE (A) and cumulative distribution of absolute MRSE (B) in subjects who received SY60CL IOL. Error bars represent $95 \%$ Cls. Abbreviations: IOL, intraocular lens; MRSE, manifest refraction spherical equivalent.

capsulotomy incidence was $1.2 \%$ at 1 year; when stratified by lens type, the incidence ranged from $0.8 \%$ to $1.8 \%$ at year $1 .{ }^{22}$

AcrySof IOLs were often reported to have among the lowest rates of PCO and capsulotomy. ${ }^{20} \mathrm{~A}$ recent metaanalysis of randomized controlled trials found that hydrophobic acrylic AcrySof IOLs had a significantly lower hazard ratio for $\mathrm{Nd}$ :YAG capsulotomy compared with other types of IOLs $(P<0.01$ for all $) .^{23}$ A prospective randomized case series in 160 eyes (2 types of IOLs implanted in contralateral eyes) reported that $\mathrm{Nd}$ :YAG capsulotomy rate was $6 \%$ for AcrySof SN60WF compared with $16 \%$ for Eyecee One (Bausch \& Lomb GmbH,
Berlin, Germany) after 3 years. ${ }^{24} \mathrm{~A}$ real-world evidence study reported that AcrySof IOLs had the lowest incidence of PCO and Nd:YAG capsulotomy rates compared with other IOLs at 3 and 5 years postoperatively (PCO rates were $4.7-18.6 \%$ and $\mathrm{Nd}: Y A G$ rates were $2.4-12.6 \%$ after 3 years; PCO rates were $7.1-22.6 \%$ and Nd:YAG capsulotomy rates were $5.8-19.3 \%$ after 5 years). ${ }^{11} \mathrm{~A}$ recent study using human capsular bag model reported similar PCO formation rates with AcrySof and Clareon IOLs. ${ }^{25}$

In this study, PCO was not assessed at the end of the cataract surgery. The rate of PCO reported in this study was $5.4 \%(19 / 350)$, and the rate of Nd:YAG capsulotomy was $4.6 \%(16 / 350)$. Although direct comparison with other IOLs 
Table 2 Nonserious and Serious AEs with Incidence $>1.0 \%$ (Safety Analysis Set)

\begin{tabular}{|l|c|}
\hline AE, $\mathbf{n}(\%)$ & SY60CL IOL (n=350) \\
\hline Posterior capsule opacification & $19(5.4)$ \\
\hline Intraocular pressure increased & $17(4.9)$ \\
\hline Punctate keratitis & $8(2.3)$ \\
\hline Vitreous detachment & $8(2.3)$ \\
\hline Dry eye & $7(2.0)$ \\
\hline Conjunctival hemorrhage & $5(1.4)$ \\
\hline Corneal edema & $4(1.1)$ \\
\hline Cystoid macular edema & $4(1.1)$ \\
\hline Blepharoplasty & $4(1.1)$ \\
\hline Iritis & $4(1.1)$ \\
\hline Visual impairment & $4(1.1)$ \\
\hline Vitreous floaters & $4(1.1)$ \\
\hline
\end{tabular}

Abbreviations: $A E$, adverse event; IOL, intraocular lens.

Table 3 Serious AEs (Safety Analysis Set)

\begin{tabular}{|l|c|}
\hline AE, $\mathbf{n}$ (\%) & SY60CL IOL (n=350) \\
\hline Cystoid macular edema & $3(0.9)$ \\
\hline Retinal laser coagulation & $2(0.6)$ \\
\hline Retinal tear & $2(0.6)$ \\
\hline Vitrectomy & $2(0.6)$ \\
\hline Corneal operation & $\mathrm{I}(0.3)$ \\
\hline Herpes virus infection & $\mathrm{I}(0.3)$ \\
\hline Macular fibrosis & $\mathrm{I}(0.3)$ \\
\hline Macular hole & $\mathrm{I}(0.3)$ \\
\hline Ophthalmologic treatment & $\mathrm{I}(0.3)$ \\
\hline Punctate keratitis & $\mathrm{I}(0.3)$ \\
\hline
\end{tabular}

Abbreviations: $A E$, adverse event; IOL, intraocular lens.

has not been done, our results suggest that the rates of $\mathrm{PCO}$ and capsulotomy for SY60CL IOLs are somewhat higher than the ranges currently reported for AcrySof IOLs. However, 6 of 16 subjects who underwent Nd:YAG in the current study were from a single site where a total of 20 subjects were enrolled, potentially distorting the interpretation of the results. The rate of capsulotomy among the remaining sites was 3\%. Additional long-term studies across multiple sites are needed to further evaluate the risks of PCO and posterior capsulotomy in subjects receiving SY60CL IOLs.

Cystoid macular edema is a common postoperative cause of visual disturbance, particularly in patients with additional risk factors such as diabetes mellitus and diabetic retinopathy. ${ }^{26,27}$ Analysis of 1659 consecutive cataract surgeries showed that the overall incidence of postoperative CME was $2.4 \% .{ }^{28}$ A retrospective database study of electronic medical records (81,984 eyes) reported that the incidence of $\mathrm{CME}$ in subjects ranged from $1.17 \%$ to $4.04 \%$ within 90 days of surgery, depending on the presence of risk factors such as diabetes. ${ }^{29}$ Other studies estimated a $0.1 \%$ to $7.0 \%$ rate of CME in subjects (including those with risk factors for CME) up to 4 months postoperatively. Additionally, assessments using fluorescein angiography and optical coherence tomography resulted in greater incidence of CME compared with CME incidence based on subjects' visual complaints. $^{30}$ A study that examined 19,980 eyes in 13,556 subjects who underwent cataract surgery in outpatient clinics in France found the 2-year incidence of CME to be $0.95 \% .{ }^{31}$ In a retrospective clinical chart review that examined real-world clinical practice records, acute $\mathrm{CME}$ incidence 3 months after cataract surgery was $0.1 \%{ }^{32}$ The CME rate of $0.9 \%$ reported in this study is consistent with other reported CME rates.

Device-related AEs reported in this study included a low rate of mild dysphotopsia $(0.9 \%)$. Dysphotopsia was reported in $32 \%$ of subjects ( $11 \%$ had severe symptoms) in a study that assessed prevalence of pseudophakic photic phenomena more than a year after the IOL implantation of Clariflex (AMO Inc, Santa Ana, CA, USA), AMO AR40e, and Alcon AcrySof IOLs. ${ }^{33}$ Those data suggest that the SY60CL IOL may have a lower prevalence of photic phenomena compared with other IOLs.

Limitations of this study included the lack of a control group, short time frame of the study, and a possible bias inherent to the controlled clinical trial setting. Further studies in subjects with SY60CL IOLs are needed to confirm long-term effects of the SY60CL material on CME, risks of PCO and posterior capsulotomy, visual disturbances, and photic phenomena. Additional studies will need to evaluate toric IOL design and address rotational stability.

In conclusion, the effectiveness of the SY60CL IOL exceeded the SPE rates for monocular CDVA, and reported safety was within the limits of the historic SPE rates. These results support the effectiveness and safety of the SY60CL IOL in correcting distance VA in aphakic subjects. 


\section{Data Sharing Statement}

The data used to support the primary findings of this study are available at clinicaltrials.gov (NCT03170154).

\section{Acknowledgments}

Medical writing assistance was provided by Natalia Zhukovskaya, PhD, of ICON (North Wales, PA), and was funded by Alcon.

\section{Funding}

This study was funded by Alcon Research LLC. Alcon assisted with the design and conduct of the study; collection, management, analysis, and interpretation of the data; and preparation, review, and approval of the manuscript.

\section{Disclosure}

RL, AM, and DML are Alcon consultants. $A F$ is a researcher and a speaker for Alcon. Dr David M Lubeck reports he is a consultant for NovaEye, consultant/investigator for Glaukos, outside the submitted work. Dr Andrew Maxwell reports personal fees from Alcon, outside the submitted work. Dr Robert Lehmann reports grants from Alcon, during the conduct of the study. The authors report no other conflicts of interest in this work.

\section{References}

1. Congdon N, Vingerling JR, Klein BE, et al. Prevalence of cataract and pseudophakia/aphakia among adults in the United States. Arch Ophthalmol. 2004;122(4):487-494.

2. Lundstrom M, Barry P, Henry Y, Rosen P, Stenevi U. Evidence-based guidelines for cataract surgery: guidelines based on data in the European Registry of Quality Outcomes for Cataract and Refractive Surgery database. J Cataract Refract Surg. 2012;38(6):1086-1093. doi:10.1016/j.jcrs.2012.03.006

3. Labuz G, Knebel D, Auffarth GU, et al. Glistening formation and light scattering in six hydrophobic-acrylic intraocular lenses. $\mathrm{Am}$ J Ophthalmol. 2018;196:112-120. doi:10.1016/j.ajo.2018.08.032

4. Hayashi K, Hirata A, Yoshida M, Yoshimura K, Hayashi H. Long-term effect of surface light scattering and glistenings of intraocular lenses on visual function. Am J Ophthalmol. 2012;152(2):240-251.e2. doi:10.1016/j.ajo.2012.03.011

5. Tognetto D, Toto L, Sanguinetti G, Ravalico G. Glistenings in foldable intraocular lenses. J Cataract Refract Surg. 2002;28(7):1211-1216. doi:10.1016/S0886-3350(02)01353-6

6. Werner L, Thatthamla I, Ong M, et al. Evaluation of clarity characteristics in a new hydrophobic acrylic IOL in comparison to commercially available IOLs. J Cataract Refract Surg. 2019;45 (10):1490-1497. doi:10.1016/j.jcrs.2019.05.017

7. Oshika T, Fujita Y, Inamura M, Miyata K. Mid-term and long-term clinical assessments of a new 1-piece hydrophobic acrylic IOL with hydroxyethylmethacrylate. J Cataract Refract Surg. 2020;46(5):682687. doi: $10.1097 /$ j.jcrs. 0000000000000142
9. Li N, Chen X, Zhang J, et al. Effect of AcrySof versus silicone or polymethyl methacrylate intraocular lens on posterior capsule opacification. Ophthalmology. 2008;115(5):830-838. doi:10.1016/j. ophtha.2007.06.037

10. Li Y, Wang J, Chen Z, Tang X. Effect of hydrophobic acrylic versus hydrophilic acrylic intraocular lens on posterior capsule opacification: meta-analysis. PLoS One. 2013;8(11):e77864.

11. Ursell PG, Dhariwal M, O'Boyle D, Khan J, Venerus A. 5 year incidence of YAG capsulotomy and PCO after cataract surgery with single-piece monofocal intraocular lenses: a real-world evidence study of 20,763 eyes. Eye (Lond). 2020;34(5):960-968. doi:10.1038/s41433-019-0630-9

12. Biber JM, Sandoval HP, Trivedi RH, et al. Comparison of the incidence and visual significance of posterior capsule opacification between multifocal spherical, monofocal spherical, and monofocal aspheric intraocular lenses. J Cataract Refract Surg. 2009;35 (7):1234-1238. doi:10.1016/j.jcrs.2009.03.013

13. Camparini M, Cassinari P, Ferrigno L, Macaluso C. ETDRS-fast: implementing psychophysical adaptive methods to standardized visual acuity measurement with ETDRS charts. Invest Ophthalmol Vis Sci. 2001;42(6):1226-1231.

14. Ophthalmic implants - intraocular lenses - part 7: clinical investigations (ISO 11979-7:2014). International Organization for Standardization; 2014.

15. Lane S, Collins S, Das K, et al. Evaluation of the mechanical behaviour of a new single-piece intraocular lens as compared to commercially available IOLs. Presented at: European Society of Cataract \& Refractive Surgeons; 2017; Lisbon, Portugal.

16. Auffarth GU, Schickhardt S, Wang Q, Fang H, Hengerer FH. Laboratory evaluation of the new Clareon hydrophobic acrylic IOL material: biomaterial properties and capsular bag behavior. Presented at: European Society of Cataract \& Refractive Surgeons; 2017; Lisbon, Portugal.

17. Nanavaty MA, Borkum S, Wedham M, et al. Clinical evaluation of visual, refractive, and adverse event outcomes of a new monofocal IOL: 6-month UK site-level results. Presented at: European Society of Cataract \& Refractive Surgeons; 2019; Paris, France.

18. Thomes BE, Callaghan TA. Evaluation of in vitro glistening formation in hydrophobic acrylic intraocular lenses. Clin Ophthalmol. 2013;7:1529-1534. doi:10.2147/OPTH.S44208

19. Schaumberg DA, Dana MR, Christen WG, Glynn RJ. A systematic overview of the incidence of posterior capsule opacification. Ophthalmology. 1998;105(7):1213-1221. doi:10.1016/S0161-6420(98)97023-3

20. Apple DJ, Peng Q, Visessook N, et al. Eradication of posterior capsule opacification: documentation of a marked decrease in $\mathrm{Nd}$ : YAG laser posterior capsulotomy rates noted in an analysis of 5416 pseudophakic human eyes obtained postmortem. Ophthalmology. 2001;108(3):505-518. doi:10.1016/S0161-6420(00)00589-3

21. Mootha VV, Tesser R, Qualls C. Incidence of and risk factors for residual posterior capsule opacification after cataract surgery. $J$ Cataract Refract Surg. 2004;30(11):2354-2358. doi:10.1016/j. jcrs.2004.03.038

22. Lindholm JM, Laine I, Tuuminen R. Five-year cumulative incidence and risk factors of Nd:YAG capsulotomy in 10044 hydrophobic acrylic 1-piece and 3-piece intraocular lenses. Am J Ophthalmol. 2019;200:218-223. doi:10.1016/j.ajo.2019.01.010

23. Thom H, Ender F, Samavedam S, et al. Effect of AcrySof versus other intraocular lens properties on the risk of Nd:YAG capsulotomy after cataract surgery: a systematic literature review and network meta-analysis. PLoS One. 2019;14(8):e0220498. doi:10.1371/journal.pone. 0220498

24. Leydolt C, Schartmuller D, Schwarzenbacher L, et al. Comparison of posterior capsule opacification development with 2 single-piece intraocular lens types. J Cataract Refract Surg. 2017;43 (6):774-780. doi:10.1016/j.jcrs.2017.06.005 
25. Hillenmayer A, Wertheimer CM, Kassumeh S, et al. Evaluation of posterior capsule opacification of the Alcon Clareon IOL vs the Alcon Acrysof IOL using a human capsular bag model. BMC Ophthalmol. 2020;20(1):77. doi:10.1186/s12886-020-013 49-5

26. Oyewole K, Tsogkas F, Westcott M, Patra S. Benchmarking cataract surgery outcomes in an ethnically diverse and diabetic population: final post-operative visual acuity and rates of post-operative cystoid macular oedema. Eye (Lond). 2017;31(12):1672-1677. doi:10.1038/ eye. 2017.96

27. Flach AJ. The incidence, pathogenesis and treatment of cystoid macular edema following cataract surgery. Trans Am Ophthalmol Soc. 1998;96:557-634.

28. Henderson BA, Kim JY, Ament CS, et al. Clinical pseudophakic cystoid macular edema. Risk factors for development and duration after treatment. J Cataract Refract Surg. 2007;33(9):1550-1558. doi:10.1016/j.jcrs.2007.05.013
29. Chu CJ, Johnston RL, Buscombe C, et al. Risk factors and incidence of macular edema after cataract surgery: a database study of 81984 eyes. Ophthalmology. 2016;123(2):316-323. doi:10.1016/j.ophtha.2015.10.001

30. Hollo G, Aung T, Cantor LB, Aihara M. Cystoid macular edema related to cataract surgery and topical prostaglandin analogs: mechanism, diagnosis, and management. Surv Ophthalmol. 2020;65 (5):496-512. doi:10.1016/j.survophthal.2020.02.004

31. Daien V, Papinaud L, Domerg C, et al. Incidence and characteristics of cystoid macular edema after cataract surgery. Ophthalmology. 2016;123(3):663-664. doi:10.1016/j.ophtha.2015.10.009

32. Packer M, Lowe J, Fine H. Incidence of acute postoperative cystoid macular edema in clinical practice. J Cataract Refract Surg. 2012;38 (12):2108-2111. doi:10.1016/j.jcrs.2012.07.029

33. Aslam TM, Gupta M, Gilmour D, Patton N, Dhillon B. Long-term prevalence of pseudophakic photic phenomena. Am J Ophthalmol. 2007;143(3):522-524. doi:10.1016/j.ajo.2006.10.031
Clinical Ophthalmology

\section{Publish your work in this journal}

Clinical Ophthalmology is an international, peer-reviewed journal covering all subspecialties within ophthalmology. Key topics include: Optometry; Visual science; Pharmacology and drug therapy in eye diseases; Basic Sciences; Primary and Secondary eye care; Patient Safety and Quality of Care Improvements. This journal is indexed on PubMed

Submit your manuscript here: https://www.dovepress.com/clinical-ophthalmology-journal
Dovepress

Central and CAS, and is the official journal of The Society of Clinical Ophthalmology (SCO). The manuscript management system is completely online and includes a very quick and fair peer-review system, which is all easy to use. Visit http://www.dovepress.com/ testimonials.php to read real quotes from published authors. 